\title{
REMARKS ON BLOW UP \\ FOR A NONLINEAR PARABOLIC EQUATION WITH A GRADIENT TERM
}

\author{
MAREK FILA
}

(Communicated by Barbara L. Keyfitz)

\begin{abstract}
We consider a nonlinear parabolic equation previously studied by Chipot and Weissler, and Kawohl and Peletier. We give simple sufficient conditions for the presence and absence of $L^{\infty}$-blow up.
\end{abstract}

\section{INTRODUCTION}

This paper was motivated by recent studies of Chipot and Weissler [CW], and Kawohl and Peletier [KP]. They studied the problem

$$
\begin{array}{ll}
u_{t}=\Delta u-|\nabla u|^{q}+\lambda u^{p}, & x \in D, t>0, \\
u(x, t)=0, & x \in \partial D, t>0, \\
u(x, 0)=u_{0}(x) \geq 0, & x \in D,
\end{array}
$$

where $D \subset \mathbb{R}^{N}$ is a smoothly bounded domain, $\lambda>0$, and $p, q>1$. Chipot and Weissler proved that blow up occurs for suitable $u_{0}$ under the assumptions

$$
1<q<2 p /(p+1), \quad p<(N+2) /(N-2) \text { if } N>2,
$$

or

$$
q=2 p /(p+1), \quad N=1, p \text { is large enough. }
$$

We give a simple sufficient condition on the initial value that implies blow up, provided $N=1, p>1$, and either $1<q<2 p /(p+1)$ or $q=2 p /(p+1)$. In the case $q=2 p /(p+1)$, we have to assume that $\lambda$ is large enough. To consider blow up as depending on the value of $\lambda$ is the point of view taken in [KP], rather than $[\mathrm{CW}]$.

A more precise description of our blow up result reads as follows. We show that $u\left(t, u_{0}\right)$ blows up if $u_{0} \geq v, u_{0} \not \equiv v$, and $v$ is the unique positive equilibrium. This is a consequence of a uniform a priori $L^{\infty}$-estimate of any global increasing solution (established also for $N>1$ ). The assumption of

Received by the editors July 19, 1989 and, in revised form, May 14, 1990.

1980 Mathematics Subject Classification (1985 Revision). Primary 35K60; Secondary 35B40.

Key words and phrases. Nonlinear parabolic equation, gradient term, blow up. 
monotonicity enables us to use a technique from $[\mathrm{F}]$ to derive the a priori bound. In [F], similar problems without gradient terms were studied.

In $[\mathrm{KP}]$ it is shown that the gradient damping term prevents blow up if $1<p \leq q=2$. We give a simple proof of the fact that blow up (in the $L^{\infty}$. norm) cannot occur if $1<p \leq q$. If, in addition, $q \leq 2$, then all solutions are global and bounded.

For $p>q=2$ one may still have $L^{\infty}$-blow up phenomena. In [KP] it is proved that given $u_{0}$ there is a $\lambda^{*}\left(u_{0}\right)>0$ such that $u\left(t, u_{0}\right)$ blows up provided $\lambda>\lambda^{*}$. We derive a lower bound for $\lambda^{*}$ in terms of the sup-norm of $u_{0}$.

\section{ESTABLISHING BLOW UP}

We adopt the notion of solution from [CW], where local theory in $W_{0}^{1, s}(D)$ was constructed ( $s$ is sufficiently large). We recall the following facts from [CW]. Assume

$$
\begin{gathered}
u_{0} \in W^{3, s} \cap W_{0}^{1, s}, \\
u_{0} \geq 0 \text { in } D, \\
\Delta u_{0}-\left|\nabla u_{0}\right|^{q}+\lambda u_{0}^{p}=0 \text { on } \partial D, \\
\Delta u_{0}-\left|\nabla u_{0}\right|^{q}+\lambda u_{0}^{p} \geq 0 \text { in } D .
\end{gathered}
$$

Then $u\left(t, u_{0}\right) \geq 0, u_{t}\left(t, u_{0}\right) \geq 0$ for all $t \in\left[0, t_{\max }\left(u_{0}\right)\right), t_{\max }\left(u_{0}\right)$ is the existence time of the maximal solution $u\left(t, u_{0}\right)$. If $q<2$ and $t_{\max }\left(u_{0}\right)<\infty$, then $\left|u\left(t, u_{0}\right)\right|_{\infty} \rightarrow \infty$ as $t \rightarrow t_{\max }\left(u_{0}\right) \quad\left(|\cdot|_{r}\right.$ denotes the norm in $L^{r}(D)$, $1 \leq r \leq \infty)$. The energy of the solution $u\left(t, u_{0}\right)$,

$$
E\left(u\left(t, u_{0}\right)\right):=\frac{1}{2}\left|\nabla u\left(t, u_{0}\right)\right|_{2}^{2}-\frac{\lambda}{p+1}\left|u\left(t, u_{0}\right)\right|_{p+1}^{p+1},
$$

is a nonincreasing function of $t \in\left[0, t_{\max }\left(u_{0}\right)\right)$ if $u_{0}$ satisfies (4)-(7). More precisely,

$$
\int_{0}^{t}\left|u_{t}(s)\right|_{2}^{2} d s+E(u(t)) \leq E\left(u_{0}\right)
$$

We are now prepared to state our first lemma.

Lemma 1. Let $u_{0}$ satisfy (4)-(7); let $t_{\max }\left(u_{0}\right)=\infty$; and assume

$$
q<2 p /(p+1), \quad \lambda>0,
$$

or

$$
q=2 p /(p+1), \quad \lambda>\bar{\lambda}_{p}=2^{p}(p+1)(p-1)^{-p-1} .
$$

Then there is a positive constant $L=L\left(E\left(u_{0}\right)\right)$ such that

$$
\left|u\left(t, u_{0}\right)\right|_{2} \leq L \quad \text { for } t \geq 0 .
$$


Proof. We proceed analogously as in the proof of Lemma 1.2 in [F]. Obvious manipulations yield that for any real number $\rho$ we have

$$
\begin{aligned}
\frac{1}{2} \frac{d}{d t}|u|_{2}^{2}= & -(p+1-\rho) E(u)+\frac{p-1-\rho}{2}|\nabla u|_{2}^{2} \\
& +\frac{\lambda \rho}{p+1}|u|_{p+1}^{p+1}-\int_{D}|\nabla u|^{q} u .
\end{aligned}
$$

Our aim is to derive a differential inequality for $y(t):=|u(t)|_{2}^{2}$. To do this we first estimate $|\nabla u|^{q} u$ using the well-known inequality:

$$
A B \leq(\varepsilon A)^{r} / r+(B / \varepsilon)^{s} / s,
$$

which holds for $A, B, r, s, \varepsilon>0,1 / r+1 / s=1$.

Take $\rho \in(0, p-1)$. Setting $A=|\nabla u|^{q}, B=u, r=2 / q$, and $\varepsilon=$ $((p-1-\rho) / q)^{q / 2}$ we obtain

$$
\begin{gathered}
|\nabla u|^{q} u \leq \frac{p-1-\rho}{2}|\nabla u|^{2}+C_{1}(p, q, \rho) u^{2 /(2-q)}, \\
C_{1}=\frac{2-q}{2}\left(\frac{p-1-\rho}{q}\right)^{q /(q-2)} .
\end{gathered}
$$

If $q<2 p /(p+1)$, then we choose $0<\delta<\lambda \rho /(p+1)$ and again we use (12) with $A=u^{2 /(2-q)}, B=C_{1}, r=(p+1)(2-q) / 2$, and $\varepsilon=(r \delta)^{1 / r}$ to get

$$
|\nabla u|^{q} u \leq \frac{p-1-\rho}{2}|\nabla u|^{2}+\delta u^{p+1}+C_{2},
$$

with $C_{2}$ being some positive constant depending on $p, q, \delta, \lambda, \rho$.

If $q=2 p /(p+1)$, then $2 /(2-q)=p+1$ and we shall need the inequality

$$
C_{1}(p, \rho)<\lambda \rho /(p+1) \text {. }
$$

To have the range of $\lambda$ minimally restricted we take $\rho=(p-1) /(p+1)$. This explains the assumption (10). Using (14) (or (13) if $q=2 p /(p+1)$ ), the inequality $E(u(t)) \leq E\left(u_{0}\right)$, and Hölder's inequality we obtain from (11) that

$$
y^{\prime} \geq C_{3} y^{(p+1) / 2}-C_{4},
$$

where $C_{3}, C_{4}$ are some positive constants that depend on $\lambda, p, q$, and $|D|-$ the Lebesgue measure of $D$, and $C_{4}$ depends also on $E\left(u_{0}\right)$. Since $u$ is a global solution and $y(t)=|u(t)|_{2}^{2}$ satisfies (15), we see that

$$
|u(t)|_{2}^{2} \leq\left(C_{4} / C_{3}\right)^{2 /(p+1)} \text { for } t \geq 0 .
$$

Lemma 2. Under the assumptions of Lemma 1 it holds that

$$
\sup _{t \geq 0}\left|u\left(t, u_{0}\right)\right|_{p+1}<\infty .
$$

Proof. Although the proof is analogous to the proof of Lemma 1.5 in [F], we shall give it here for the reader's convenience. It is based on the classical concavity method introduced in [L]. Assume that $\left|u\left(t, u_{0}\right)\right|_{p+1}$ is unbounded. Then

$$
\left|u\left(t, u_{0}\right)\right|_{p+1} \rightarrow \infty \text { as } t \rightarrow \infty
$$

since $\left|u\left(\cdot, u_{0}\right)\right|_{p+1}$ is nondecreasing. 
Put

$$
M(t)=\int_{0}^{t}|u|_{2}^{2}
$$

If $q<2 p /(p+1)$ we use (14) and (8) to derive the following inequality from (11):

$$
\frac{1}{2} M^{\prime \prime}(t) \geq(p+1-\rho)\left(\int_{0}^{t} \int_{D} u_{t}^{2}-E\left(u_{0}\right)\right)+\left(\frac{\lambda \rho}{p+1}-\delta\right)|u(t)|_{p+1}^{p+1}-|D| C_{\delta} .
$$

Writing

$$
\begin{aligned}
M^{\prime 2}(t) & =\left(\int_{0}^{t} \frac{d}{d t}|u|_{2}^{2}+\left|u_{0}\right|_{2}^{2}\right)^{2} \\
& =4\left(\int_{0}^{t} \int_{D} u u_{t}\right)^{2}+2 M^{\prime}\left|u_{0}\right|_{2}^{2}-\left|u_{0}\right|_{2}^{4}
\end{aligned}
$$

we obtain

$$
\begin{aligned}
M M^{\prime \prime} & -\frac{p+1-\rho}{2} M^{\prime 2} \\
\geq & 2(p+1-\rho)\left(\int_{0}^{t} \int_{D} u^{2} \int_{0}^{t} \int_{D} u_{t}^{2}-\left(\int_{0}^{t} \int_{D} u u_{t}\right)^{2}\right) \\
& +2 M\left(\left(\frac{\lambda \rho}{p+1}-\delta\right)|u(t)|_{p+1}^{p+1}-(p+1-\rho) E\left(u_{0}\right)-|D| C_{\delta}\right) \\
& -(p+1-\rho) M^{\prime}\left|u_{0}\right|_{2}^{2}+(p+1-\rho)\left|u_{0}\right|_{2}^{4} / 2 .
\end{aligned}
$$

The first term on the right-hand side is nonnegative according to the Schwarz inequality; the second one tends to infinity by (16); and the third one is bounded according to Lemma 1 . Hence, there is a $t_{0} \geq 0$ such that the right-hand side is positive for $t \geq t_{0}$. Therefore $\left(M^{-\mu}\right)^{\prime \prime}<0$ for $t \geq t_{0}$, where $\mu=$ $(p+1-\rho) / 2>0$. Since $M^{-\mu}$ is decreasing, it must have a root, a contradiction.

If $q=2 p /(p+1)$ we proceed similarly. The only difference is that we use (13) instead of (14).

Theorem 1. Let (4)-(7) and (9) or (10) hold. Assume further that $t_{\max }\left(u_{0}\right)=$ $\infty$ and

$$
p<(N+2) /(N-2) \text { if } N>2 .
$$

Then there is a constant $K=K\left(\left|u_{0}\right|_{\infty}, E\left(u_{0}\right)\right)$ such that $\left|u\left(t, u_{0}\right)\right|_{\infty} \leq K$ for $t \geq 0$. Moreover, $u\left(t, u_{0}\right) \rightarrow v$ in $W_{0}^{1, s}(D)$ as $t \rightarrow \infty ; v$ is a stationary solution.

Proof. If $u$ is a nonnegative function that satisfies $u_{t} \leq \Delta u+\lambda u^{p} \quad$ in $D \times(0, \infty), \quad u=0 \quad$ on $\partial D \times(0, \infty), \quad u(\cdot, 0) \in L^{\infty}(D)$, 
then it is known (see e.g. [R, Proposition 2]) that boundedness of $u$ in $L^{r}$ implies boundedness in $L^{\infty}$ provided $r>(p-1) \max \{1, N / 2\}$. More precisely,

$$
\left|u\left(t, u_{0}\right)\right|_{\infty} \leq C\left(\left|u_{0}\right|_{\infty}, \sup _{0 \leq s \leq t}\left|u\left(s, u_{0}\right)\right|_{r}\right) \text { for } t \geq 0 .
$$

Lemma 2 and (18) with $r=p+1$ yield that

$$
\sup _{t \geq 0}\left|u\left(t, u_{0}\right)\right|_{\infty}<\infty \text {. }
$$

For $q \leq 2$, we have the estimate

$$
\|u\|_{C^{1+\mu,(1+\mu) / 2}(\bar{D} \times[0, T])} \leq \gamma\left(\|u\|_{C(\bar{D} \times[0, T])},\left\|u_{0}\right\|_{C^{2}(\bar{D})}\right)
$$

for any $\mu \in(0,1)$ and $T>0$ (see [A, Theorem 2.2]). Therefore, $\left\{u\left(t, u_{0}\right)\right.$ : $t \geq 0\}$ is precompact in $W_{0}^{1, s}(D)$. Due to monotonicity $u\left(t, u_{0}\right) \rightarrow v, v$ is a stationary solution.

Similarly as in the proof of Lemma 1.8 in [F], we derive an a priori bound of $|v|_{p+1}$ in terms of $E\left(u_{0}\right)$. For $v$ it holds that

$$
|\nabla v|_{2}^{2}=\lambda|v|_{p+1}^{p+1}-\int_{D}|\nabla v|^{q} v
$$

therefore,

$$
\begin{aligned}
(p+ & 1-\rho) E\left(u_{0}\right) \\
& >(p+1-\rho) E(v)=\frac{p-1-\rho}{2}|\nabla v|_{2}^{2}+\frac{\lambda \rho}{p+1}|v|_{p+1}^{p+1}-\int_{D}|\nabla v|^{q} v \\
& \geq(\lambda \rho /(p+1)-\delta)|v|_{p+1}^{p+1}-|D| C_{\delta} \quad \text { if } q<2 p /(p+1) \\
& \left(\geq\left(\lambda \rho /(p+1)-C_{1}\right)|v|_{p+1}^{p+1} \quad \text { if } q=2 p /(p+1) .\right)
\end{aligned}
$$

The last inequality follows from (14) (or (13)).

The monotonicity of $\left|u\left(\cdot, u_{0}\right)\right|_{p+1}$ together with (18) now implies the desired result.

Before we state the next theorem, let us recall some known results (proved in [CW]) on the one-dimensional stationary problem. If (9) holds or if $q=$ $2 p /(p+1), \lambda>\lambda_{p}=(2 p)^{p}(p+1)^{-2 p-1}$ (notice that $\left.\bar{\lambda}_{p}>\lambda_{p}\right)$, then there is a unique positive stationary solution $v$ (for any interval $D$ ). From Lemma 4.7 and Proposition 5.9 in [CW], it follows that it is unstable, because it is possible to construct supersolutions arbitrarily close to $v$ from below and subsolutions arbitrarily close to $v$ from above.

Theorem 2. Let (9) or (10) hold. Assume that $N=1, u_{0} \in W_{0}^{1, s}(D), u_{0} \geq v$, and $u_{0} \not \equiv v$, where $v$ is the unique positive stationary solution. Then $u\left(t, u_{0}\right)$ blows up in a finite time (in the $L^{\infty}$-norm).

Proof. Since $v$ is unstable, there is a solution $w$ of $(1),(2)$ on $\bar{D} \times(-\infty, 0]$ such that $w_{t}>0, w>v$ in $D \times(-\infty, 0]$ and $w \rightarrow v$ in $C^{2}(\bar{D})$ as $t \rightarrow$ $-\infty\left(\right.$ see $\left[\mathrm{M}\right.$, Theorem 1]). Hence $t_{\max }(w(\cdot, 0))<\infty$; otherwise $u(t, w(\cdot, 0))$ 
would tend to a stationary solution $z>v$ (by Theorem 1). The maximum principle implies the existence of $t_{1}<0 \leq t_{2}$ for which $w\left(\cdot, t_{1}\right) \leq u\left(t_{2}, u_{0}\right)$ and the conclusion follows.

\section{EXCLUDING BLOW UP}

Theorem 3. Assume that $u_{0} \in W_{0}^{1, s}(D), u_{0} \geq 0$.

(i) If $q \geq p>1$, then there is a constant $K_{1}=K_{1}\left(\left|u_{0}\right|_{\infty}\right)>0$ such that $u\left(t, u_{0}\right) \leq K_{1}$ for $t \in\left[0, t_{\max }\left(u_{0}\right)\right)$.

(ii) If $p>q \geq 2 p /(p+1)$, then there are positive numbers $\tilde{\lambda}=\tilde{\lambda}\left(\left|u_{0}\right|_{\infty}\right)$, $K_{2}=K_{2}\left(\left|u_{0}\right|_{\infty}\right)$ such that $u\left(t, u_{0}\right) \leq K_{2}$ for $t \in\left[0, t_{\max }\left(u_{0}\right)\right)$, provided $0<\lambda<\tilde{\lambda}$.

Proof. Suppose first for simplicity that $N=1, D=(1, a)$, and $a>1$. Then

$$
U(x):=\alpha^{2 /(p-1)} e^{\alpha x}
$$

is a suitable supersolution.

In the case (i) it suffices to choose

$$
\alpha=\max \left\{\left|u_{0}\right|_{\infty}^{(p-1) / 2},(1+\lambda)^{\beta}\right\}, \quad \beta=\frac{p-1}{q(p+1)-2 p} .
$$

In the case (ii) take

$$
\alpha=\max \left\{1,\left|u_{0}\right|_{\infty}^{(p-1) / 2}\right\}, \quad \tilde{\lambda}=e^{a \alpha(q-p)}\left(\alpha^{1 / \beta}-e^{\alpha(1-q)}\right) .
$$

In both cases we have

$$
\begin{aligned}
& U^{\prime \prime}(x)-\left(U^{\prime}(x)\right)^{q}+\lambda U^{p}(x) \\
& \quad=e^{\alpha p x b} \alpha^{2 p /(p-1)}\left(e^{\alpha(1-p) x}-\alpha^{1 / \beta} e^{\alpha(q-p) x}+\lambda\right) \leq 0 ;
\end{aligned}
$$

hence by the maximum principle $u\left(t, u_{0}\right) \leq U$ for $0 \leq t<t_{\max }\left(u_{0}\right)$.

For a general domain $D$ in $\mathbb{R}^{N}$, we set

$$
U(x)=\alpha^{2 /(p-1)} \exp \left(\alpha \sum_{i=1}^{N} x_{i}\right)
$$

and we may assume without loss of generality that $\sum_{i=1}^{N} x_{i} \in(1, a)$ for $x=$ $\left(x_{1}, \ldots, x_{N}\right) \in D$.

Remark. If $q \leq 2$, then $t_{\max }\left(u_{0}\right)=\infty$ in Theorem 3, due to (19). This implies that $u\left(t, u_{0}\right) \rightarrow 0$ as $t \rightarrow \infty$ for any $u_{0} \in W_{0}^{1, s}, u_{0} \geq 0$, provided $1<p \leq q \leq$ 2 and $D$ is a ball with a sufficiently small radius. Under these circumstances, no positive stationary solutions exist (see [CW, Corollary 5.4 (i)]).

\section{ACKNOWLEDGMENTS}

This paper was written while the author was visiting the University of Heidelberg. This visit was supported by the Deutsche Forschungsgemeinschaft. The author wishes to thank B. Kawohl for his interest, and the referee for his valuable comments. 


\section{REFERENCES}

[A] H. Amann, Existence and multiplicity theorems for semi-linear elliptic boundary value problems, Math. Z. 150 (1976), 281-295.

[CW] M. Chipot and F. B. Weissler, Some blow up results for a nonlinear parabolic equation with a gradient term, SIAM J. Math. Anal. 20 (1989), 886-907.

[F] M. Fila, Boundedness of global solutions of nonlinear diffusion equations, J. Differential Equations (to appear).

[KP] B. Kawohl and L. A. Peletier, Observations on blow up and dead cores for nonlinear parabolic equations, Math. Z. 202 (1989), 207-217.

[L] H. Levine, Some nonexistence and instability theorems for solutions of formally parabolic equations of the form $P u_{t}=-A u+F(u)$, Arch. Rational Mech. Anal. 51 (1973), 371-386.

[M] H. Matano, Existence of nontrivial unstable sets for equilibriums of strongly order preserving systems, J. Fac. Sci. Tokyo 30 (1983), 645-673.

[R] F. Rothe, Uniform bounds from bounded $L_{p}$-functionals in reaction-diffusion equations, $J$. Differential Equations 45 (1982), 207-233.

Department of Mathematical Analysis, Comenius University, Mlynská dolina, 84215 Bratislava, Czechoslovakia 